\title{
COVID-19 und die Ökonomik von Leben und Tod
}

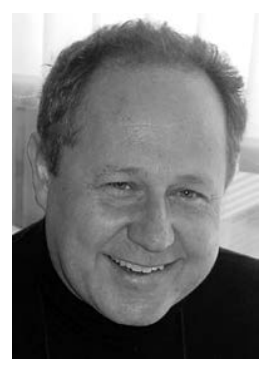

Manfred Wildner

\author{
Bibliografie \\ Gesundheitswesen 2021; 83: 324-326 \\ DOI 10.1055/a-1467-9678 \\ ISSN $0941-3790$ \\ (C) 2021. Thieme. All rights reserved. \\ Georg Thieme Verlag KG, Rüdigerstraße 14, \\ 70469 Stuttgart, Germany
}

Korrespondenzadresse

Prof. Dr. med. Manfred Wildner

Pettenkofer School of Public Health

c/o Bayerisches Landesamt für

Gesundheit und Lebensmittelsicherheit

Veterinärstraße 2

85764 Oberschleißheim

Deutschland

Manfred.Wildner@lgl.bayern.de
Im Jahr 1993 veröffentlichte Amartya Sen seinen nicht nur in Fachkreisen beachteten Artikel „The Economics of Life and Deaths“ [1]. In diesem Beitrag, ins Deutsche zu übersetzen als „Wirtschaftswissenschaftliche Überlegungen [Economics] zu Leben und Tod“, formuliert der Harvard-Professor und spätere Nobel-Preisträger wesentliche Gedanken seiner kritischen Wohlfahrtsökonomik auf dem Weg zu einer „Ökonomie für den Menschen“ [2]. Er weist darauf hin, dass sich die Wirtschaftswissenschaften nicht nur mit Ressourcen wie Einkommen und Vermögen als solchen beschäftigen sollten, sondern auch mit deren Gebrauch zu bedeutsameren Zwecken, insbesondere für ein langes und lebenswertes Leben. Amartya Sen illustriert seine Überlegungen in dem o.g. Beitrag u. a. mit der Hungersnot des Jahres 1974 in Bangladesh. Auslöser der Hungersnot war damals kein Ausfall bei der tatsächlichen Reisernte, sondern ein Ineinandergreifen verschiedener anderer Faktoren und ein ungenügendes staatliches Handeln. Am Anfang standen zunächst Überflutungen von Juni bis August 1974 mit zeitweisen Auswirkungen auf die Reisproduktion, welche in der Folge zur Arbeitslosigkeit von Tagelöhnern in den betroffenen Regionen führten. Ohne Einkommen fehlten deren Familien die Mittel zum Kauf benötigter Nahrung - eine sich daran anschließende allgemeine Panik führte zu Hamsterkäufen und Vorratsbildungen durch die finanziell leistungsstärkeren Bevölkerungsgruppen mit wiederum in der Folge stark steigenden Lebensmittelpreisen. Der Teufelskreis war in Gang gesetzt: Der Höhepunkt der Lebensmittelpreise im Oktober des Jahres 1974 korrelierte eng mit der Höhe der Sterberate bei den mittellosen Bevölkerungsgruppen. Gegenmaßnahmen der Regierung unterblieben zunächst durch die vordergründig beruhigenden „nackten“ Zahlen einer an sich ausreichenden Nahrungsmittelverfügbarkeit. Die verspätete Regierungsreaktion mit Beschaffung von Nahrungsmitteln aus den USA wurde wiederum durch politische Ressentiments auf US-Seite wegen Jutelieferungen aus Bangladesh nach Kuba verzögert. Letztlich hatte die Hungersnot ihre vielen Opfer bei den einkommensschwachen Bevölkerungsteilen bereits gefordert, bevor die letztlich überaus gute Ernte des Jahres 1974 eingebracht wurde. Amartya Sen schlägt dazu verschiedene Gegenmaßnahmen vor: Eine vorausschauende staatliche Inzentivierung der erforderlichen Produktion und darüber hinaus eine wirtschaftliche Diversifizierung, öffentliche Beschäftigungsprogramme bei Arbeitslosigkeit als Alternative zu unproduktiven Lagerbildungen mit dort notwendigen Lebensmittelverteilungen und insbesondere auch eine demokratische Verfassung mit freier Presse, um notwendiges rasches Handeln zu unterstützen.

Für eine allgemein erstrebenswerte menschliche Entwicklung mit bestmöglichen Verwirklichungschancen („Capability Approach“) ist nach Sens Ansicht das ökonomische „Arrangement“ einer Gesellschaft zentral. Für dieses Arrangement sind wiederum mehrere Freiheiten essentiell: Die politische Freiheit, wie sie durch demokratische Verfassungen am besten gewährleistet werden, eine sozial regulierte freie Marktwirtschaft, eine Gewährleistung der Chancengerechtigkeit, eine dazu gehörende soziale Absicherung und ganz wesentlich auch gesellschaftliche Transparenz. Letztere dient insbesondere auch als Vorbeugung gegenüber einem missbräuchlichen Umgang mit den vorhandenen Ressourcen und damit verbunden mit Macht.

Der Bezug zu COVID-19 und unserem Gesundheitswesen? Ein solcher Bezug mag zunächst verwundern, sind doch die Gesellschaften der Europäischen Union vergleichsweise wohlhabend und mit leistungsfähigen Gesundheitssystemen ausgestattet, insbesondere auch in Deutschland. Und doch bringt die Pandemie der Jahre 2020/21 vieles auf den Punkt, was an die andauernde Armut anderer Gesellschaften anknüpfen lässt. Zunächst die relative Knappheit medizinischer Ressourcen: Der Ressource der Intensiv- 
betten in der Krankenversorgung im Besonderen, deren Verknappung durch die solidarische Kraftanstrengung mehrerer gesamtgesellschaftlicher Lock-downs und partieller wirtschaftlicher Shutdowns kurzfristig erfolgreich begegnet wurde - mittel- und längerfristige Herausforderungen in der Pflege bleiben allerdings ungelöst. Dann die Knappheit der Impfstoffe, welche wiederum solidarisch auf Ebene der Europäischen Union durch gemeinsame Einkäufe und europaweite Verteilungen in gegebenen Grenzen erfolgreich begegnet wurde, ergänzt durch eine variable Priorisierung und Binnenverteilung innerhalb der EU-Länder. Diese Maßnahmen wurden in unterschiedlichem Ausmaß transparent diskutiert und durch öffentliche Berichterstattung begleitet. Die bisweilen hitzige Diskussion in Deutschland sollte nicht nur kritisch gewertet werden, sondern auch als unvermeidliche Folge bzw. notwendige Begleitung einer transparenten Entscheidungsfindung, welche gemeinschaftliche Erfolge wie auch politisches Versagen jeweils beim Namen nennen kann.

Die mit Daten und Geld erkauften raschen Impfprogramme kleiner Staaten stellen hierzu keine Alternative dar, auch nicht die isolierte „we first“-Mentalität ehemaliger oder aktueller Supermächte. Und auch die vordergründigen Erfolge von Staaten mit riskanten Beschaffungsprogrammen wie in Chile sollten noch genauer betrachtet werden: Der dort beschaffte und auch vertragsmäßig gelieferte Impfstoff aus China hat nach vorliegenden ersten Auswertungen nach der ersten Impfung eine Effektivität von lediglich $3 \%$, nach einer zweiten Impfung von etwas über $50 \%$ - Erfolge der Durchimpfung relativieren sich damit in ihrer Schutzwirkung dann doch etwas [3]. Andere Impfstoffe aus China haben bessere Leistungsdaten [4], auch für den Impfstoff Sputnik V aus Russland liegen grundsätzlich gute Leistungsdaten vor [5], auch wenn die Qualitätsprüfungen erfolgter Lieferungen außerhalb Russlands zu heftiger Kritik geführt haben. Insgesamt wurden bahnbrechende Ergebnisse mit den Impfstoffen gegen COVID-19 erarbeitet, in Europa und den USA mit den neuen mRNA-Impfstoffen ebenso wie mit hoch wirksamen herkömmlichen Vektor-Impfstoffen, mit Abstrichen auch in China, Russland und in Indien - ein Erfolg von Marktwirtschaft als System und marktwirtschaftlicher Diversifizierung, allerdings wesentlich auch mit staatlicher Paten- und Partnerschaft, verbunden mit sozialer Regulierung und auch mit dem notwendigen Korrektiv einer regulierten Marktüberwachung.

Weniger transparent verliefen diverse andere Beschaffungsmaßnahmen, von Schutzmasken bis zu Testsystemen - wohl nicht nur, aber auch in Deutschland. Hier sollte die von Amartya Sen ebenfalls geforderte Transparenz nachdrücklich und nachhaltig eingefordert werden. Transparenz auf Seiten der staatlichen Verwaltung und der politischen Mandatsträger, welche auf die Verfassungstreue, auf die Beachtung von Recht und Gesetz vereidigt wurden. Damit unter ethischen Gesichtspunkten nur schlecht vereinbar sind Beschaffungsverträge für Schutzmasken, welche mandatierten parlamentarischen Abgeordneten Zahlungen in Millionenhöhe bescheren. Eine „schöne Bescherung“: Der Schaden für die demokratischen Institutionen und die Demokratie als politisches System ist immens. Auch nur schlecht mit Recht und Gesetz vereinbar sind wirtschaftsseitige Lieferungen von Schutzgütern, bei denen die Konformität mit bestehenden Qualitätsvorgaben offenbar leichterdings bestätigt wird, welche sich dann bei Überprüfungen aber als nicht verkehrsfähig herausstellen - dieser Betrug am Bürger kos- tet nicht nur Geld und Vertrauen, sondern auch Leben. Diesem Vorgehen gleichgestellt sind Leistungsangaben auch renommierter Hersteller von Schnelltest-Systemen, welche sich bei Überprüfungen als bei weitem überhöht herausstellen. Auch solches Agieren kostet Geld, Vertrauen und Menschenleben [6]. Unseriöse JubelPublikationen sollten insbesondere in Krisen auf Leben und Tod sträflich sein, notwendig in einem wörtlichen Sinn sind verlässliche Daten und daran anknüpfende, rationale und wissenschaftlich begründete und transparent kommunizierte Strategien [7]. Hinsichtlich der unverzichtbaren neutralen und objektiven Rolle höchster staatlicher Ämter und staatlicher Überwachungsbehörden bei der Beschaffung und Zulassung solcher Schnelltestsysteme stellen sich unangenehme Fragen, sollten sich Pressemeldungen zu unrechtmäßigen Einflussnahmen und Nepotismen erhärten [8, 9]. Eine Reform insbesondere der Medizinproduktezulassung und -überwachung wird seit längerem gefordert [10]. In der Summe ist einmal mehr festzuhalten, dass menschliche Gemeinschaft in einem freiheitlichen, säkularisierten Staat von Voraussetzungen lebt, welche dieser Staat selbst nicht garantieren kann (Ernst-Ludwig Böckenförde). Diese Gemeinschaft lebt damit von einem sozialen Kapital, welches sich wesentlich auch in nicht-staatlichen Sozialisierungen und im weiteren auch persönlichen Entscheidungen und Rückbindungen begründet. Ausdrucksformen sind u. a. charakterliche Verlässlichkeit und Gesetzestreue, Mitgefühl und Fairness, Gemeinsinn und eine gemeinschaftliche Begrenzung und Kontrolle von Macht und auch ein ernsthaftes Streben nach Wahrheit in der Erkenntnis der Wirklichkeit.

Einem solchen Streben nach Wahrheit und Erkenntnis sind die Beiträge dieses Heftes verpflichtet: Zur Gesundheitskompetenz in Deutschland während der digitalen Aufholjagd, zu Charakteristika von durch den Innovationsfonds geförderten Interventionsstudien, zu Angebotsstrukturen der ambulanten vertragsärztlichen Versorgung mit zugehörigem Kommentar, zu Strategien eines risikostratifizierten Einsatzes von Antigen-Schnelltests, zur Nachverfolgung der COVID-19 Pandemie über Google-Sucheingaben, zur statistischen Untererfassung von Norovirus-Infektionen, zur Asiatischen Tigermücke (Aedes albopictus) als Herausforderung für den Öffentlichen Gesundheitsdienst, zur Situation der angestellten Ärztinnen und Ärzte im ambulanten Sektor in Deutschland, zu PFLEGEprevent, einem Präventivprojekt, um der Arbeitsbelastung von Pflegekräften begegnen, zum Zusammenhang von chronischen Straßenlärm-Expositionen und dem Blutdruck (Metaanalyse) sowie zu den Auswirkungen lärmmindernder Maßnahmen auf die Häufigkeit von Lärmbelästigung, Schlafstörungen und Herz-KreislaufErkrankungen als Modellrechnung sowie, als Beitrag zur Aufarbeitung einer herausfordernden Vergangenheit, zu Männern in geschlossenen Venerologischen Stationen der DDR am Beispiel von Berlin, Dresden und Erfurt.

Um am Ende noch einmal zum Anfang zurückzukehren: Im Zusammenhang mit der Corona-Pandemie wird deutlich, dass die wirtschaftlichen Zusammenhänge von (Über-)Leben und Tod nicht auf Entwicklungsländer und auch hierzulande nicht auf frühere Zeiten begrenzt sind. „Politik beginnt mit dem Betrachten der Wirklichkeit“ (Kurt Schumacher) - auch wenn dies vielleicht erst ein Desiderat ist: Teile dieser zu betrachtenden Wirklichkeit wären zum einen die Sterblichkeitsziffern, wie dies Amartya Sen in dem eingangs zitierten Beitrag fordert. In Zusammenhang mit der COVID- 
19 Pandemie werden solche Betrachtungen in der Aufarbeitung notwendig werden: Hinsichtlich der Rationalität, Effektivität und Effizienz der angewendeten Maßnahmen, hinsichtlich der Disparitäten ihrer Auswirkungen auf unterschiedliche gesellschaftliche Gruppen, hinsichtlich der gesundheitlichen Folgen von Betrug und Vorteilsnahme, unseriöser Produktinformationen und daran anknüpfender hemdsärmeliger Schutzmaßnahmen und hinsichtlich notwendiger verbesserter Steuerungsinformationen für die politischen Staatenlenker. Denkbar und für die Zukunft notwendig sind auch grundsätzliche gesellschaftskritische Reflexionen zu Fairness und Solidarität, innerhalb staatlicher Grenzen und auch staatlicher Institutionen und dann auch weit darüber hinaus in einer dem Geschehen angemessenen internationalen und globalen Sichtweise. Dies wird sich zum anderen aber absehbar nicht allein auf die - oft späten - Sterbeziffern gründen lassen, sondern darauf aufbauend auch aktuelle Steuerungsinformationen aus verschiedenen Lageperspektiven und auch in ihren sozialen Schichtungen und Verteilungen - zur epidemischen Dynamik und zur medizinischen Versorgung, zu den gesundheitlichen Folgen auch bei den (Über-)Lebenden und zu den bestehenden Handlungsoptionen - benötigen. Wir schwimmen im selben pandemischen Strom, sitzen aber nicht im gleichen Boot. Vielleicht lassen sich diese Perspektiven, anstelle einer einzelnen summierenden „Zahl“ zur Lagebewertung, in Anlehnung an das betriebswirtschaftliche Instrument einer verschiedene Aspekte integrierenden „Balanced Scorecard“ [11] zukünftig zusammenstellen als eine differenzierte und auch sozial balancierte „Scorecard für Gesundheit“. Damit wiederum anknüpfend an die von Amartya Sen in den Mittelpunkt gestellten menschlichen Verwirklichungschancen, die von den Vereinten Nationen im Index für die menschliche Entwicklung [12] aufgegriffen wurden und zu welchem Gesundheit etwa ein Drittel beiträgt: Als wesentlicher Beitrag für das Leben - auch nach einer Pandemie.

\section{Interessenkonflikt}

Die Autorinnen/Autoren geben an, dass kein Interessenkonflikt besteht.
Literatur

[1] Sen A. The Economics of Life and Deaths. Scientific American 1993; 266: $40-47$

[2] Sen A. Ökonomie für den Menschen. Wege zu Gerechtigkeit und Solidariät in der Markswirtschaft. München, Hanser 2000 [engl. Original: Development as Freedom. New York: Oxford University Press; 1999

[3] Chile Covid-19 vaccination drive adds to Sinovac efficacy data. https:// www.scmp.com/news/china/science/article/3128886/chile-covid19-vaccination-drive-adds-sinovac-efficacy-data accessed 2021-04-10

[4] What do we know about China's covid-19 vaccines? BM] 2021; 373 : $\mathrm{n} 912$

[5] Logunov DY, Dolzhikova IV, Shcheblyakov DV et al for the Vaccine Trial Group. Safety and efficacy of an rAd26 and rAd5 vector-based heterologous prime-boost COVID-19 vaccine: an interim analysis of a randomised controlled phase 3 trial in Russia. Lancet 2021; 397: 671-681

[6] Denkinger CM, Brümmer LE. Antigen Schnelltests für die Diagnose einer SARS CoV2 Infektion. https://diagnosticsglobal health.org/ accessed 2021-04-10

[7] Stratil JM, Arnold L (2021): Strategie zum risikostratifizierten Einsatz von Antigen-Schnelltests: Eindämmung der SARS-CoV-2-Pandemie durch die Integration von Schnelltests in das Fall-und Kontaktpersonenmanagement. Gesundheitswesen 2021; in Druck. Siehe auch: https://www.public-health-covid19.de/images/2021/Ergebnisse/210302_Kompetenznetz_Schnellteststrategie_final2.pdf accessed 2021-04-10

[8] Bayerischer PCR-Schnelltest erhält Sonderzulassung. https://www. aerzteblatt.de/nachrichten/119746/Bayerischer-PCR-Schnelltesterhaelt-Sonderzulassung accessed 2021-04-10

[9] Weitere Vorwürfe gegen CSU-Abgeordneten Alfred Sauter. https:// www.zeit.de/politik/deutschland/2021-04/korruptionsaffaere-alfredsauter-csu-schnelltests-vorwuerfe-korruption?utm_referrer $=$ https \%3A\%2F \%2Fwww.google.de \%2F accessed 2021-04-10

[10] Reinhardt D, Wildner M. Inverkehrbringen von Arzneimitteln und Medizinprodukten in Deutschland: Evaluation der Verfahren und Schwachstellenanalyse. Gesundheitswesen 2016; 78: 844-852

[11] Kaplan RS, Norton DP. The Balanced Scorecard - Measures that Drive Performance. Harvard Business Review, Januar/Februar 1992

[12] Anand S, Sen A. Human Development Index: Methodology and Measurement. Hrsg.: Entwicklungsprogramm der Vereinten Nationen, Human Development Report Office (= Occasional Papers. Nr. 12). New York Juli. 1994; URL http://hdr.undp.org/sites/default/files/oc12.pdf, accessed 2021-04-10])Originalarbeit 